\title{
Nonlinear optical interactions of topological modes of photonic nanostructures
}

Lan, Zhihao, You, Jian Wei, Panoiu, Nicolae

Zhihao Lan, Jian Wei You, Nicolae Panoiu, "Nonlinear optical interactions of topological modes of photonic nanostructures," Proc. SPIE 11461, Active

Photonic Platforms XII, 114610X (6 October 2020); doi: 10.1117/12.2568438

SPIE. Event: SPIE Nanoscience + Engineering, 2020, Online Only 


\title{
Nonlinear optical interactions of topological modes of photonic nanostructures
}

\author{
Nicolae C. Panoiu \\ Department of Electronic and Electrical Engineering, University College London, Torrington \\ Place, London WC1E 7JE, United Kingdom
}

\begin{abstract}
Topological photonics aims to utilize topological photonic bands and corresponding edge modes to implement robust light manipulation. Importantly, topological photonics provide an ideal platform to study nonlinear interactions. In this paper, some recent results regarding nonlinear interactions of one-way edge-modes in frequency mixing processes in topological photonic nanostructures are reviewed. More specifically, we will discuss the band topology of $2 \mathrm{D}$ photonic crystals with hexagonal symmetry and demonstrate that second-harmonic generation (SHG) and third-harmonic generation can be implemented via one-way edge modes. In addition, four-wave mixing of topological plasmon modes of graphene plasmonic crystals and SHG upon interaction of valley-Hall topological modes of all-dielectric photonic crystals will be discussed.
\end{abstract}

Keywords: Topological photonics, nonlinear optics, valley-Hall topological modes, graphene

\section{INTRODUCTION}

A key development in condensed matter physics in recent years has been the discovery of topological insulating materials. ${ }^{1,2}$ These materials feature gapped bulk and gapless edge modes, which propagate unidirectionally along the system surface, thus providing opportunities for robust wave manipulation protected by topology. Related to these developments, topological photonics aims to extend these ideas to the realm of photonics, ${ }^{3-9}$ which holds great promises for new optical devices by exploiting robust, scattering-free light propagation and control. As the concept of energy band exists at the single particle level both in condensed matter physics and photonics, the goal of realizing photonic topological insulators can be easily achieved in the linear optics. Indeed, many topological phenomena of electromagnetic waves in a linear medium can be understood by mapping Maxwell and Schrödinger equations. ${ }^{10,11}$

Photonics, however, has several features not present in solid-state physics. For instance, optical gain and loss can be utilized to implement non-Hermitian photonics based on parity-time symmetry. ${ }^{12}$ Another well-known feature is the existence of nonlinearity in many optical materials, which gives rise to a variety of important phenomena, including the formation of solitons, modulation and all-optical switching of optical signals, and frequency conversion for the generation of ultrashort pulses. ${ }^{13}$ Thus one expects new physics to emerge when adding nonlinearity to photonic systems with nontrivial topological properties. Indeed, it has been demonstrated that lattice edge solitons arise ${ }^{14,15}$ when a photonic topological insulator is embedded in an optical medium with Kerr nonlinearity. The possibility to enhance the conversion efficiency of harmonic generation in the presence of topological edge states has also been studied. ${ }^{16-18}$ Moreover, traveling-wave amplifiers, ${ }^{19}$ topological sources of quantum light, ${ }^{20}$ nonlinear control ${ }^{21}$ and mapping ${ }^{22}$ of photonic topological edge states have also been achieved.

Valley degree of freedom, which is associated to the conduction-band minima (or valence-band maxima) in graphene-like two-dimensional (2D) materials, ${ }^{23}$ has recently been introduced to photonics, ${ }^{24}$ too. These 2D materials exhibit nontrivial Berry curvature distribution in the momentum space around each valley, which gives rise to a valley-dependent topological index associated to the integral of Berry curvature around a valley. Furthermore, a domain-wall interface separating two topologically distinct valley photonic crystals (PhCs) can support valley-momentum locked modes localized at the interface, similar to the quantum-valley Hall effect. ${ }^{24}$ Until recently, valley-Hall photonic modes have been mostly studied in bulk materials, being less explored

\footnotetext{
Nicolae C. Panoiu: E-mail: n.panoiu@ucl.ac.uk
} Vol. 11461, 114610X · @ 2020 SPIE · CCC code: 0277-786X/20/\$21 · doi: 10.1117/12.2568438 
in $2 \mathrm{D}$ photonic platforms, including graphene. ${ }^{25,26}$ This $2 \mathrm{D}$ material is becoming a promising platform to achieve passive and active topologically protected plasmonic modes ${ }^{27-29}$ due to its high carrier mobility and long relaxation time. ${ }^{30,31}$

This invited paper is organized as follows. In the next section we present the physical properties of nonlinear interaction of topological modes of PhCs and use second- and third-harmonic generation (SHG, THG) as illustrative examples. Then, in Sec. 3, we discuss the four-wave mixing (FWM) of topological modes of graphene metasurfaces, whereas in Sec. 4 the topic of SHG via interaction of topological valley-Hall kink modes is addressed. Finally, in the last section we summarize the main conclusions and present some perspectives on future developments in this rapidly evolving area of research.

\section{NONLINEAR INTERACTION OF TOPOLOGICAL MODES OF PHOTONIC CRYSTALS}

In this section we present the main features of the nonlinear interaction of topological modes of a certain class of PhCs. ${ }^{32}$ Thus, we consider triangular PhCs whose unit cell contains a cylinder with radius, $r$, as depicted in Fig. 1(a). Lattice structures with hexagonal symmetry but having more cylinders in each unit cell, like honeycomb and Kagome lattices with two and three cylinders, respectively, could potentially be employed, too. The second step of our design procedure is to include magnetic and nonlinear materials. To guide potential experimental implementations, we consider cylinders with low-permittivity $\left(\epsilon_{1}\right)$, non-magnetic nonlinear material immersed in a magnetic background material with high-permittivity $\left(\epsilon_{2}\right)$. Note that the permittivity of the cylinders has to be lower than that of the background to ensure that Dirac cones exist.

We then consider the topological properties of the bulk frequency bands of the non-magnetic $\left(\mu=\mu_{0}\right) \mathrm{PhCs}$ whose first Brillouin zone is shown in Fig. 1(b). Figure 1(c) shows the photonic band structure of the PhC with $r=0.4 a, \epsilon_{1}=3$, and $\epsilon_{2}=18$. From this figure one can see that there is a Dirac cone between the first and second
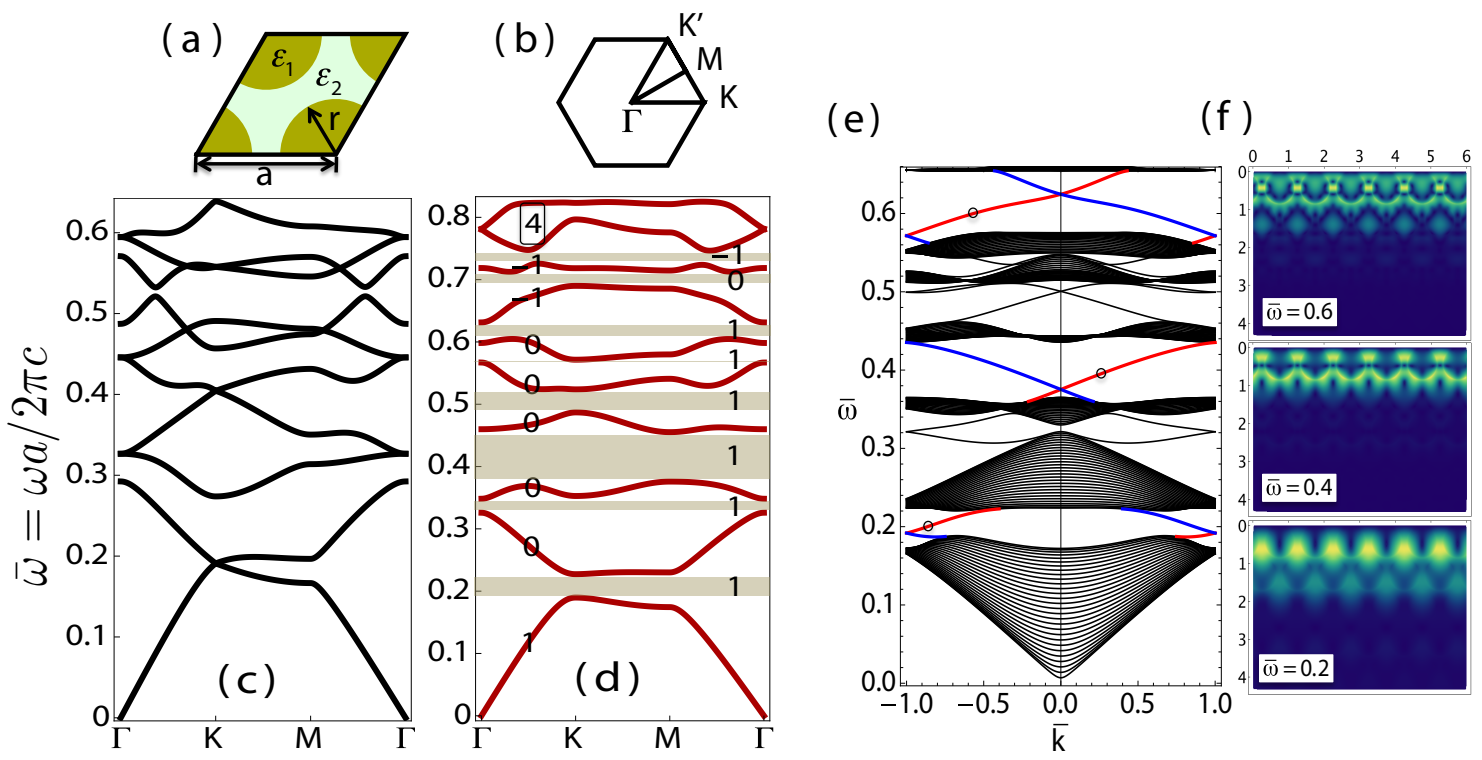

Figure 1. (a) The unit cell with lattice constant $a$ of the $\mathrm{PhC}$, where $r$ and $\epsilon_{1}$ are the radius and relative permittivity of the cylinders, respectively, and $\epsilon_{2}$ and $\mu_{i}$ are the relative permittivity and off-diagonal component of the relative permeability of the background magnetic material, respectively. (b) The first Brillouin zone with the symmetry points, $\Gamma, K, K^{\prime}$, and $M$. (c) Photonic band structure of the PhC, computed for $r=0.4 a, \epsilon_{1}=3, \epsilon_{2}=18$, and $\mu_{i}=0$. (d) Nontrivial topological bands for $\mu_{i}=0.8$, where the Chern number of each band and the gap Chern numbers are provided. (e) Photonic band structure of a $1 \mathrm{D} \mathrm{PhC} \mathrm{strip} \mathrm{that} \mathrm{is} \mathrm{periodic} \mathrm{along} \mathrm{the} x$-axis and has finite size of 30 unit cells along the $y$-axis. The other simulation parameters are $\epsilon_{1}=3, \epsilon_{2}=20$, and $\mu_{i}=0.8$. The edge modes in the three gaps around $\bar{\omega}=0.2,0.4$, and 0.6 are depicted by red and blue lines and are formed at the top and bottom edges of the PhC, respectively. (f) Field profiles of the three one-way edge modes at $\bar{\omega}=0.2,0.4$, and 0.6 . 
bands at $K$ and $K^{\prime}$ points. The Chern number of each band is zero in systems with time-reversal symmetry, ${ }^{33}$ so such systems are topologically trivial. A common way to break time-reversal symmetry and generate bands with nonzero Chern number is to use magnetic materials, where the permeability tensor of the material under an external magnetic field along the $z$-axis possesses off-diagonal components in the $x-y$ plane,

$$
\mu=\left(\begin{array}{ccc}
\mu_{0} & i \mu_{i} & 0 \\
-i \mu_{i} & \mu_{0} & 0 \\
0 & 0 & \mu_{0}
\end{array}\right)
$$

Here, we set $\mu_{0}=1$ and take $\mu_{i}$ as a parameter to quantify the effect of time-reversal symmetry breaking. Figure 1(d) shows the band structure for $\mu_{i}=0.8$, where one can see that the Dirac cone is gapped.

In the next step of our design of a $\mathrm{PhC}$ suited to investigate the nonlinear interaction of topological modes, we choose the proper parameters to create photonic gaps suitable to study SHG and THG. According to the principle of bulk-edge correspondence in systems with finite size, when the gap has nonzero Chern number, one-way edge modes will emerge in the gap. We present in Fig. 1(e) the photonic band structure of a PhC strip with width of 30 unit cells and periodic along the longitudinal direction. This figure illustrates the emergence of various edge states across bulk photonic gaps. For clarity, we mark in red and blue the edge states formed on the top and bottom edges of the $\mathrm{PhC}$ strip, respectively. The field profiles of the edge states at frequencies $\bar{\omega}=0.2, \bar{\Omega}_{2}=2 \bar{\omega}=0.4$, and $\bar{\Omega}_{3}=3 \bar{\omega}=0.6$, presented in Fig. 1(f), highlight the key feature of the edge state, namely the exponential decay of the field away from the edge.

The full-wave dynamics of the nonlinear interaction of topological edge-modes (SHG and THG) were determined numerically using COMSOL Multiphysics. Thus, we defined two "Electromagnetic Waves, Frequency Domain" models, one for the fundamental frequency $\omega_{0}$ and one for the second (third) harmonic frequency $\Omega_{2}$ $\left(\Omega_{3}\right)$. We assumed that for both the SHG and THG cases the nonlinear susceptibilities are diagonal tensors, the diagonal elements being $\chi_{2}$ and $\chi_{3}$, respectively. In the case of the SHG, the nonlinear polarizations at the FF and $\mathrm{SH}$ are: ${ }^{34} P_{\mathrm{NL}, z}^{\omega_{0}}=2 \chi_{2} E_{2 z} E_{1 z}^{*}$ and $P_{\mathrm{NL}, z}^{\Omega_{2}}=\chi_{2} E_{1 z}^{2}$, respectively, whereas for the THG they are: ${ }^{34}$ $P_{\mathrm{NL}, z}^{\omega_{0}}=3 \chi_{3} E_{3 z} E_{1 z}^{*}{ }^{2}$ and $P_{\mathrm{NL}, z}^{\Omega_{3}}=\chi_{3} E_{1 z}^{3}$, respectively. In the following, we mainly focus on the discussion of SHG, as the results of THG can be understood similarly.

We consider cylinders made of homogeneous and isotropic nonlinear material with nonlinear second-order susceptibility of $\chi^{(2)}=10^{-21} \mathrm{CV}^{-2}$. The pump electric field $\mathbf{E}_{1}$ is induced by an external source whereas $\mathbf{E}_{2}$ by the nonlinear polarization at the SH. From Figs. 2(a), one can observe that the field profiles of $\mathbf{E}_{1}$ and $\mathbf{E}_{2}$ are indeed the same as the profiles of the edge modes shown in Fig. 1(f), indicating that the two edge modes are indeed nonlinearly interacting. The physics of this nonlinear process can be captured by the period of spatial oscillations of the $\mathrm{SH}$ field $\mathbf{E}_{2}$, which is determined by the wave-vector mismatch $\Delta k=k_{S H}-2 k_{F F}$
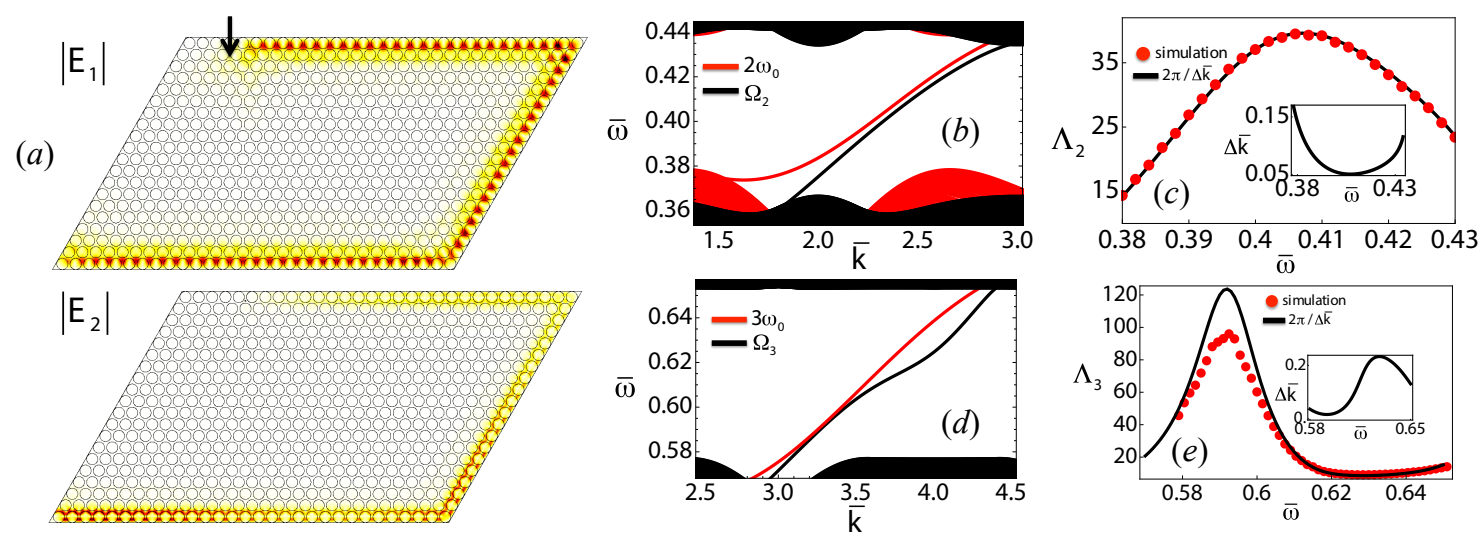

Figure 2. (a) Field profile intensities $\mathbf{E}_{1}$ and $\mathbf{E}_{2}$ at $\bar{\omega}=0.2$ and $\bar{\Omega}_{2}=2 \bar{\omega}=0.4$, respectively. The SHG is simulated with $\chi^{(2)}=10^{-21} \mathrm{CV}^{-2}$. (b) The edge modes at FF $\omega_{0}$ (plotted in terms of $2 \omega_{0}$ ) and for the SHG, $\Omega_{2}$. (c) Theoretically calculated $\Lambda_{2}=2 \pi / \Delta \bar{k}$ using CMT and numerically extracted oscillation period of $\mathbf{E}_{2}$. (d), (e) The results corresponding to (b), (c), respectively, but calculated for the THG. 
$\left(\Delta k=k_{T H}-3 k_{F F}\right.$ for THG). As a result, we can compare the numerically extracted oscillation period of $\mathbf{E}_{2}$ with the theoretical prediction of $\Lambda_{2}=2 \pi / \Delta \bar{k}$, thus confirming that the key physics of nonlinear frequency conversion processes is validated by our simulations.

These conclusions were further validated using a much larger simulation domain, the corresponding results being presented in Figs. 2(b) and 2(c). It can be seen that there is an excelent agreement between the predictions of the coupled-mode theory ${ }^{32}$ (CMT) and direct numerical simulations. We calculated $\Delta \bar{k}$ for all the frequencies of the interacting edge modes from Fig. 2(b) and present the theoretically calculated and numerically extracted oscillation period $\Lambda_{2}$ in Fig. 2(c). An excellent agreement between the two results can be observed, which confirms that phase matching is indeed at work in our photonic system and SHG purely via nonlinear interaction of edge modes occurs. We also confirm the edge mode mediated THG as shown in Figs. 2(d) and 2(e), where the discrepancy in the latter figure between the numerical and theoretical results is due to the limitations of numerical simulations at very small $\Delta k$.

\section{FOUR-WAVE MIXING OF TOPOLOGICAL MODES OF GRAPHENE METASURFACES}

The nonlinear system considered in this section is illustrated in Fig. 3(a), and comprises of a graphene plasmonic metasurface consisting of a hexagonal periodic nanohole array with lattice constant $a$ and air hole radius $r$ placed in a static magnetic field. Due to the time-reversal-symmetry breaking induced by the magneto-optical response of graphene under an external magnetic field, this plasmonic system could possess a topological bandgap. In order to induce a FWM process, ${ }^{29}$ the system is excited by an external source at the pump frequency $\omega_{p}$. Due to the strong third-order nonlinearity of graphene, a degenerate FWM process could take place, where two photons in a pump mode will generate a pair of photons at the signal and idler frequencies, $\omega_{s}$ and $\omega_{i}$, respectively. As a result, the energy of the pump mode is transferred to the signal and idler modes, leading to the pump decay and the amplification of the signal and idler. More importantly, this degenerate FWM process is topologically protected by the chiral nature of the edge plasmons.

The projected band diagrams along $k_{x}$, determined for $B=0$ and $B=5 \mathrm{~T}$, are presented in Fig. 3(b). It can be seen that a bandgap opens when one applies an external static magnetic field $(B \neq 0)$. Moreover, in the band diagrams of Fig. 3(b) there are two edge modes at the top (red) and bottom (blue) boundaries of the finite graphene system. These two edge modes connect the bulk bands located above and below the bandgap, and cannot be moved out of the bandgap into the bulk bands as long as the bandgap exists. In other words,
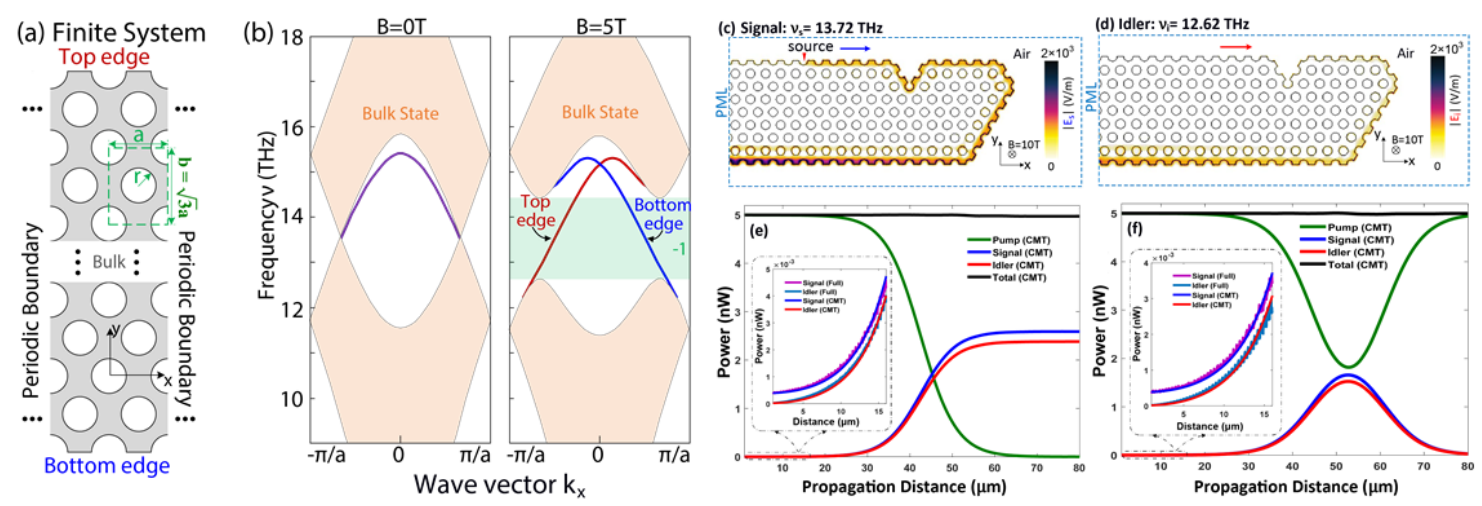

Figure 3. (a) Geometry of the finite graphene metasurface, where the number of unit cells (green dashed frame) is finite along the $y$-axis and infinite along the $x$-axis. (b) Projected band diagrams of the metasurface at $B=0$ and $B=5 \mathrm{~T}$, where the edge modes on the top and bottom boundaries are depicted by red and blue curves, respectively. (c) The field profile at the signal frequency, $\nu_{s}=13.72 \mathrm{THz}$. (d) The field profile at the idler frequency, $\nu_{i}=12.62 \mathrm{THz}$. (e) Dependence on the propagation distance of the mode power of the pump, signal, and idler corresponding to the field profiles shown in (c) and (d), determined using the CMT when the FWM process is phase-matched. Also shown in the insets are the same mode powers determined using the CMT and full-wave simulations. (f) The same as in (e), but corresponding to a case when the FWM interaction is not phase-matched. 
they are robust and defect-immune. Since the gap Chern number is -1 (the magnitude indicates the number of topological edge modes, whereas the sign shows the direction of propagation), there is only one topologically protected edge mode for each edge termination.

The dynamics of the FWM process and its main physical properties are summarized in Figs. 3(c)-3(f). Note that in these calculations graphene losses are neglected, but the overall qualitative picture remains unchanged when they are added. It can be seen in Figs. 3(c) and 3(d) that, as a result of the nonlinear FWM interaction, the signal is amplified upon propagation whereas an edge mode is generated at the idler frequency. Moreover, since the frequencies of all the interacting edge modes are located in the topological bandgap, both signal and idler modes are topologically protected and exhibit unidirectional propagation along the system edge. The FWM process can be further, quantitatively investigated by calculating the dependence on the propagation distance of the power carried by the three edge modes. The results of these calculations are summarized in Figs. 3(e) and $3(\mathrm{f})$, and correspond to the case of near phase-matching and a case when the FWM process is not phase-matched, respectively. In the latter case, the system parameters are $\nu_{p}=13.03 \mathrm{THz}, \nu_{s}=14.05 \mathrm{THz}, \nu_{i}=12.01 \mathrm{THz}$, and $\Delta \kappa=1.75 \times 10^{-2}$.

There are several important ideas revealed by the results presented in Figs. 3(e) and 3(f). First, the power of both the signal and idler modes is amplified upon propagation, due to the energy conversion from the pump mode. Second, the growth rate of signal and idler modes in the case of the nearly phase-matched FWM is larger than when the FWM interaction is not phase matched, which means that the energy conversion is more efficient in the former case. Third, the plots presented in the insets of Figs. 3(e) and 3(f) clearly show that the predictions of the CMT agree very well with the rigorous results obtained using full-wave simulations, despite the fact that the optical fields at the three frequencies are strongly confined at deep-subwavelength scale.

\section{SECOND-HARMONIC GENERATION VIA INTERACTION OF TOPOLOGICAL VALLEY-HALL MODES}

To study the SHG via interaction of topological valley-Hall modes, we consider a $2 \mathrm{D}$ honeycomb PhC made of dielectric cylinders with radius $r_{1}$ and $r_{2}$, as per Fig. 4(a), whose unit cell is shown in Fig. 4(b). The cylinders are made of nonlinear material with dielectric constant $\epsilon$ and second-order nonlinear susceptibility $\chi^{(2)}$. The transverse magnetic modes of the honeycomb PhC possess Dirac points between the first and second bands. ${ }^{35} \mathrm{In}$ Fig. 4(c) we show the corresponding band structure of the PhC, determined for $r_{1}=r_{2}$, from which one can see the existence of double Dirac points. In an all-dielectric implementation, the inversion symmetry can be broken by using a unit cell containing cylinders with different radius. We show in Fig. 4(d) the band structure of the $\mathrm{PhC}$ with $r_{1}=0.18 a$ and $r_{2}=0.22 a$, from which one can see the gapping out of the Dirac points by forming valley gaps. The band structure of a kink-type domain wall interface is presented in Fig. 4(e). The two sets of kink modes, depicted in red and blue, correspond to the two ways to construct the interface. One can see that, the kink modes within the two valley gaps have opposite slopes at a specific valley.

For convenience of illustrating the SHG, we present both the fundamental and second-harmonic kink modes in the second valley gap, see Fig. 4(f). A complication in the current all-dielectric $\mathrm{PhC}$ structure stems from the fact that, as the system has time-reversal symmetry, at each frequency there are two kink modes (corresponding to the two valleys at $K$ and $K^{\prime}$ ) for both the fundamental and the second-harmonic components. Although one can excite the fundamental wave unidirectionally exploiting the inherent chirality of the kink modes, e.g., by using sources of either right- or left-circularly polarized light, ${ }^{25,26}$ generally the generated second-harmonic waves will have both forward- and backward-propagating components. A typical example is shown in Fig. 4(g), where the three kink modes participating in the nonlinear process and the frequency are indicated in Fig. 4(f) by dots and the blue line, respectively. While the fundamental wave at $\bar{k}_{f}$ is launched unidirectionally towards right (with the source marked by the arrow), from the field intensity of the harmonic wave $\left|E_{2}\right|$, one can see that both forward- and backward-propagating waves are generated.

\section{CONCLUSION}

In conclusion, we have presented topology-protected nonlinear frequency conversion processes via one-way edge modes of topological photonic crystals. In addition, we discussed the physics of a topologically protected nonlinear 

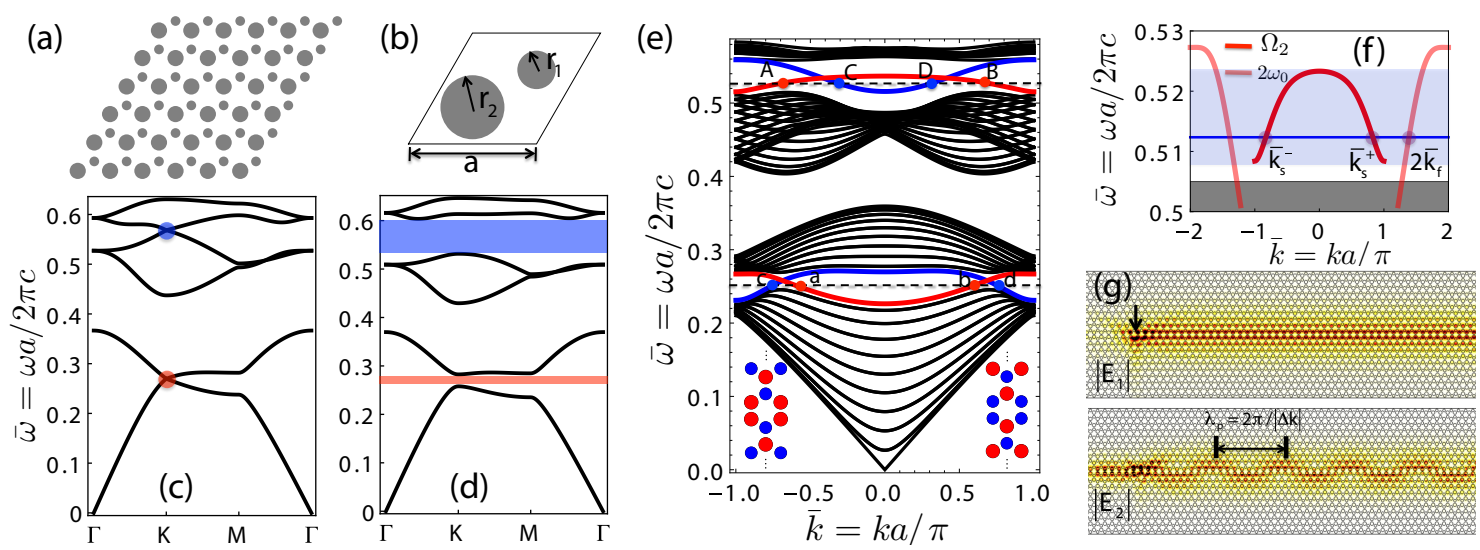

Figure 4. (a) Schematic of the system. (b) Unit cell of the $\mathrm{PhC}$, containing two cylinders of nonlinear material with radius $r_{1}, r_{2}$, dielectric constant $\epsilon=12$ and $\chi^{(2)}=10^{-21} \mathrm{CV}^{-2}$. (c) The existence of double Dirac points (marked by red and blue dots) of the PhC at $r_{1}=r_{2}=0.2 a$. (d) The same as in (c), but calculated for $r_{1}=0.18 a$ and $r_{2}=0.22 a$. (e) Band structure of a kink-type domain wall interface separating two PhCs with $r_{1}=0.2 a, r_{2}=0.23 a$, which are mirror symmetric to each other (see the inserts). (f) Dispersion curves used for the SHG, determined for $r_{1}=0.19 a$ and $r_{2}=0.24 a$. Shaded grey area corresponds to bulk modes whereas the light blue indicates the frequency matching window. (g) Intensities of the fundamental $\left(E_{1}\right)$ and second-harmonic $\left(E_{2}\right)$ waves at the frequency marked by the blue line in (f).

four-wave mixing process in a graphene metasurface, showing that a topological bandgap as wide as several terahertz can be created in the metasurface under a strong static magnetic field. Moreover, the analysis of the dispersion properties of the topologically protected edge modes revealed that four-wave mixing interaction is efficiently phase matched in a large domain of the parameter space of the system. Finally, we have also illustrated bi-directional second-harmonic generation via nonlinear interaction of topological valley-Hall kink modes within two valley gaps in all-dielectric photonic crystals. Importantly, other nonlinear interactions of topological modes, such as sum- and difference-frequency generation, high-harmonic generation, and cross-phase modulation, can be readily implemented within the photonic platforms discussed here.

\section{ACKNOWLEDGMENTS}

This work has been supported by the European Research Council (ERC), Grant no. ERC-2014-CoG-648328.

\section{REFERENCES}

[1] M. Z. Hasan and C. L. Kane, Colloquium: Topological insulators, Rev. Mod. Phys. 82, 3045 (2010).

[2] X.-L. Qi and S.-C. Zhang, Topological insulators and superconductors, Rev. Mod. Phys. 83, 1057 (2011).

[3] L. Lu, J. D. Joannopoulos, and M. Soljacic, Topological photonics, Nat. Photonics 8, 821 (2014).

[4] A. B. Khanikaev and G. Shvets, Two-dimensional topological photonics, Nat. Photonics 11, 763 (2017).

[5] X.-C. Suna, C. He, X.-P. Liu, M.-H. Lu, S.-N. Zhu, and Y.-F. Chen, Two-dimensional topological photonic systems, Prog. Quantum. Electron. 55, 52 (2017).

[6] Y. Wu, C. Li, X. Hu, Y. Ao, Y. Zhao, and Q. Gong, Applications of Topological Photonics in Integrated Photonic Devices, Adv. Opt. Mater. 5, 1700357 (2017).

[7] B.-Y. Xie, H.-F. Wang, X.-Y. Zhu, M.-H. Lu, Z. D. Wang, and Y.-F. Chen, Photonics meets topology, Opt. Express 26, 2453 (2018).

[8] M. S. Rider, S. J. Palmer, S. R. Pocock, X. Xiao, P. A. Huidobro, and V. Giannini, A perspective on topological nanophotonics: Current status and future challenges, J. Appl. Phys. 125, 120901 (2019).

[9] T. Ozawa, H. M. Price, A. Amo, N. Goldman, M. Hafezi, L. Lu, M. C. Rechtsman, D. Schuster, J. Simon, O. Zilberberg, and I. Carusotto, Topological Photonics, Rev. Mod. Phys. 91, 015006 (2019).

[10] F. D. M. Haldane and S. Raghu, Possible Realization of Directional Optical Waveguides in Photonic Crystals with Broken Time-Reversal Symmetry, Phys. Rev. Lett. 100, 013904 (2008). 
[11] G. D. Nittis and M. Lein, On the role of symmetries in the theory of photonic crystals, Ann. Phys. 350, $568(2014)$.

[12] L. Feng, R. EI-Ganainy, and L. Ge, Non-Hermitian photonics based on parity-time symmetry, Nat. Photonics 11, 752 (2017).

[13] R. W. Boyd, Nonlinear Optics, Academic Press; 3 edition (2008).

[14] Y. Lumer, Y. Plotnik, M. C. Rechtsman, and M. Segev, Self-Localized States in Photonic Topological Insulators, Phys. Rev. Lett. 111, 243905 (2013).

[15] D. Leykam and Y. D. Chong, Edge Solitons in Nonlinear-Photonic Topological Insulators, Phys. Rev. Lett. 117, 143901 (2016).

[16] C. Qian, K. H. Choi, R. P. H. Wu, Y. Zhang, K. Guo, and K. H. Fung, Nonlinear frequency up-conversion via double topological edge modes, Opt. Express 26, 5083 (2018).

[17] Y. Wang, L.-J. Lang, C. H. Lee, B. Zhang, and Y. D. Chong, Topologically enhanced harmonic generation in a nonlinear transmission line metamaterial, Nat. Commun. 10, 1102 (2019).

[18] S. Kruk, A. Poddubny, D. Smirnova, L. Wang, A. Slobozhanyuk, A. Shorokhov, I. Kravchenko, B. L.Davies, and Y. S. Kivshar, Nonlinear light generation in topological nanostructures, Nat. Nanotechnol. 14, 126 (2019).

[19] V. Peano, M. Houde, F. Marquardt, and A. A. Clerk, Topological Quantum Fluctuations and Traveling Wave Amplifiers, Phys. Rev. X 6, 041026 (2016).

[20] S. Mittal, E. A. Goldschmidt, and M. Hafezi, A topological source of quantum light, Nature 561, 502 (2018).

[21] D. A. Dobrykh, A. V. Yulin, A. P. Slobozhanyuk, A. N. Poddubny, and Y. Kivshar, Nonlinear Control of Electromagnetic Topological Edge States, Phys. Rev. Lett. 121, 163901 (2018).

[22] D. Smirnova, S. Kruk, D. Leykam, E. M.-Gaykazyan, D.-Y. Choi, and Y. Kivshar, Third-Harmonic Generation in Photonic Topological Metasurfaces, Phys. Rev. Lett. 123, 103901 (2019).

[23] D. Xiao, W. Yao, and Q. Niu, Valley-contrasting physics in graphene: magnetic moment and topological transport, Phys. Rev. Lett. 99, 236809 (2007).

[24] T. Ma and G. Shvets, All-Si valley-Hall photonic topological insulator, New J. Phys. 18, 025012 (2016).

[25] Y. Wang, J. W. You, Z. Lan, and N. C. Panoiu, Topological valley plasmon transport in bilayer graphene metasurfaces for sensing applications, Opt. Lett. 45, 3151-3154 (2020).

[26] J. W. You, Z. Lan, Q. Bao, and N. C. Panoiu, Valley-Hall topological plasmons in a graphene nanohole plasmonic crystal waveguide, IEEE J. Sel. Top. Quantum Electron. 26, 4600308 (2020).

[27] D. Jin, T. Christensen, M. Soljacic, N. X. Fang, L. Lu, and X. Zhang, Infrared topological plasmons in graphene, Phys. Rev. Lett. 118, 245301 (2017).

[28] J. W. You and N. C. Panoiu, Tunable and dual-broadband giant enhancement of second-harmonic and third-harmonic generation in an optimized graphene-insulator-graphene metasurface, Phys. Rev. B 102, 121403(R) (2020).

[29] J. W. You, Z. Lan, and N. C. Panoiu, Four-wave mixing of topological edge plasmons in graphene metasurfaces, Sci. Adv. 6, eaaz3910 (2020).

[30] J. W. You, S. Bongu, Q. Bao, and N. C. Panoiu, Nonlinear optical properties and applications of 2D materials: theoretical and experimental aspects, Nanophotonics 8, 63-97 (2018).

[31] P. A. D. Goncalves and N. M. Peres, An Introduction to Graphene Plasmonics (World Scientific, 2016).

[32] Z. Lan, J. W. You, and N. C. Panoiu, Nonlinear one-way edge-mode interactions for frequency mixing in topological photonic crystals, Phys. Rev. B 101, 155422 (2020).

[33] Z. Wang, Y. D. Chong, J. D. Joannopoulos, and M. Soljacic, Reflection-Free One-Way Edge Modes in a Gyromagnetic Photonic Crystal, Phys. Rev. Lett. 100, 013905 (2008).

[34] N. C. Panoiu, W. E. I. Sha, D. Y. Lei, and G.-C. Li, Nonlinear optics in plasmonic nanostructures, J. Opt. 20, 083001 (2018).

[35] X.-D. Chen, F.-L. Zhao, M. Chen, and J.-W. Dong, Valley-contrasting physics in all-dielectric photonic crystals: Orbital angular momentum and topological propagation, Phys. Rev. B 96, 020202(R) (2017). 